\title{
ON THE $n$-PARAMETER CONCORDANCE AND ISOTOPY THEOREM
}

\author{
TADATOSHI AKIBA ${ }^{1}$
}

\begin{abstract}
J. F. P. Hudson [4] proved that "concordance" implies "isotopy". In this paper we show that the $n$-parameter version of Hudson's result cannot be true without some restrictions. Assuming Millett's result [6], we can determine a specific dimension where the $n$-parameter version fails. We rely on Kan fibrations and geometric techniques such as the Alexander trick.
\end{abstract}

The purpose of this paper is two-fold. One is to present a theorem which will be a basis for a forthcoming paper [2]. The other is to show that the semisimplicial complex $\mathcal{E}\left(I \times L, I \times M_{m}\right.$ rel $\left.U\right)$ is not contractible, which is a corollary of our theorem by assuming Millett's result [6].

Although our main theorem will be generalized in a future paper, the present paper is written separately because the generalization depends on the results of this paper, and the method used there does not simplify the present situation.

We adopt notations in [1] and assume the reader's familiarity with basic works such as [5] and [7]. We work in the piecewise linear (PL) category exclusively, and all maps, embeddings, etc. are understood to be PL.

Let $M_{m}=\mathrm{Cl}\left(D^{n}-\cup_{i=1}^{m} D_{i}^{n}\right)$, where $D_{i}^{n}$ 's are mutually disjoint convex $n$ cells in Int $D^{n}$. The manifold $M_{0}=D^{n}$. Let $L$ be a convex 1-cell connecting a point $p$ of $\partial D^{n}$ to another $p_{m}$ of $\partial D_{m}^{n}$ without intersecting any Int $D_{i}^{n}$. Unless otherwise specified, we assume $n>2$ in the rest for simplicity, although most of the results are valid without this restriction.

Let $U \subset \partial\left(I \times M_{m}\right)$ be the submanifold $I \times\left\{p, p_{m}\right\} \cup 0 \times L$.

THEOREM. If $\pi_{i}\left(\varepsilon\left(I \times L, I \times M_{m}\right.\right.$ rel $\left.\left.U\right)\right) \cong 0$ for all $i \leqq k$ and all $m$, then $\pi_{k+2}\left(\mathcal{E}_{\partial}\left(I, I \times S^{n}\right)\right) \cong \pi_{k+3}\left(S^{n}\right)$.

Assuming the results of Millett [6, Theorem 1.17] we can conclude

Corollary. $\pi_{2 n-5}\left(\mathcal{E}\left(I \times L, I \times M_{m}\right.\right.$ rel $\left.\left.U\right)\right) \nRightarrow 0$ when $\pi_{2 n-2}\left(F_{n}, G_{n}\right) \cong 0$.

This provides the first example where the homotopy group of

Received by the editors December 30, 1974 and, in revised form, November 18, 1975. AMS (MOS) subject classifications (1970). Primary 57C35; Secondary 55E20.

Key words and phrases. $k$-isotopy, semisimplicial complex, Alexander trick, fibration.

${ }^{1}$ The author wishes to thank the referee for helpful suggestions. 


$$
\mathcal{E}\left(I \times L, I \times M_{m} \operatorname{rel} U\right)
$$

is nontrivial.

The rest of the paper will be devoted to a proof of the theorem. We prove two propositions which, together, imply the theorem. When $P \subset M_{m}$ is a subpolyhedron, we denote $I \times P$ by $P^{1}$. We often identify $I \times a$ with $I$ (and $0 \times a$ with $a$ ) for a distinguished point $a \in \operatorname{Int} M_{m}$.

Proposition 1. If $\pi_{i}\left(\varepsilon\left(L^{1}, M_{m}^{1} \operatorname{rel} U\right)\right) \cong 0$ for all $i \leqq k$ and $m$, then $\pi_{i}\left(\mathcal{E}\left(I, I \times M_{m}\right.\right.$ rel 0$\left.)\right) \cong 0$ for all $i \leqq k+1$ and $m$.

Proposition 2. If $\pi_{i}\left(\mathscr{E}\left(I, I \times M_{m}\right.\right.$ rel 0$\left.)\right) \cong 0$ for all $i \leqq p$ and $m$, then $\pi_{i}\left(\mathcal{E}_{\partial}\left(I, I \times S^{n}\right)\right) \cong \pi_{i+1}\left(S^{n}\right)$ for all $i \leqq p+1$.

1. Proof of Proposition 1. By a cell we always mean a convex cell. Let $H \subset L$ be a one-cell such that $H \cap \partial M_{m}=\{p\}(\partial H=\{p, q\}), V=I \times \partial H$ $\cup 0 \times H$, and $N$ a subinterval of $H-\{p\}$ containing $q$. Also $C=I \times q \cup 0$ $\times N$.

LEMMA 3. The following are Kan fibrations, where $r$ is the restriction:

$$
\mathcal{E}\left(H^{1}, M_{m}^{1} \operatorname{rel} V\right) \hookrightarrow \mathcal{E}\left(H^{1}, M_{m}^{1} \operatorname{rel} 0 \times H \cup I \times p\right)
$$

$$
\stackrel{r}{\longrightarrow} \mathcal{E}\left(I \times q, M_{m}^{1} \operatorname{rel} 0 \times q\right) .
$$

$$
\mathcal{E}\left(H^{1}, M_{m}^{1} \operatorname{rel} V \cup N^{1}\right) \hookrightarrow \mathcal{E}\left(H^{1}, M_{m}^{1} \operatorname{rel} V\right) \stackrel{r}{\rightarrow} \mathcal{E}\left(N^{1}, M_{m}^{1}, \operatorname{rel} C\right) .
$$

This lemma follows from Lemma 1 of [1].

Note that the total space of $(\mathrm{a})$ is contractible by the next lemma.

LEMMA 4. Let $K$ be an $i$-disk in a q-manifold $Q(i \leqq q)$ and $U$ a neighborhood of $K$ in $Q$ such that $(U, K) \approx\left(D^{1} \times D^{i-1} \times D^{q-i}, I \times D^{l-1} \times 0\right)$. Then

$$
\mathcal{E}(K, Q \operatorname{rel}(K \cap \partial Q))
$$

is contractible.

The proof is an easy application of the Alexander trick, (see [5, 3A], for example) and is omitted here.

Definition. A $k$-modification of $M$ with respect to $\mathcal{E}(K, M$ rel $Y)$ is a $(k+1)$-homeotopy $F \in \mathcal{H}(M$ rel $Y)$ such that $F \mid 0 \times \Delta_{k} \times M \cup I \times \partial \Delta_{k} \times K$ $=$ identity. $F_{t}$ will stand for $F \mid t \times \Delta_{k} \times M$. When there is no confusion, we omit mentioning $M$ and $\varepsilon(K, M$ rel $Y)$.

Lemma 5. The natural inclusion $i: \varepsilon\left(N^{1}, M_{m}^{1} \operatorname{rel} C\right) \rightarrow \mathcal{E}\left(N^{1}, M_{0}^{1}\right.$ rel $\left.C\right)$ is a homotopy equivalence for all $m \geqq 0$.

Proof. Let $f$ represent an element $[f]$ of $\pi_{k}\left(\mathscr{E}\left(N^{1}, M_{0}^{1}\right.\right.$ rel $\left.\left.C\right)\right)$. We show that $[f]=i_{*}[g]$, for some $g \in \mathcal{E}\left(N^{1}, M_{m}^{1} \operatorname{rel} C\right)$. 
First, shrink $f\left(\Delta_{k} \times 1 \times N\right)$ toward $f\left(\Delta_{k} \times 1 \times q\right)$, using $\operatorname{Im} f$ as the trace of the $k$-modification $F$ so that the end result $f^{\prime}=F_{1} \circ f$ satisfies

$$
f^{\prime}(x \times 1 \times N) \cap x \times 1 \times \partial M_{m}=\varnothing .
$$

Next, apply a similar argument to $f^{\prime}\left(\Delta_{k} \times I \times N\right)$ so that the end result $f^{\prime \prime}$ of a $k$-modification satisfies

$$
f^{\prime \prime}\left(\Delta_{k} \times N^{1}\right) \cap \Delta_{k} \times I \times \partial M_{m}=\varnothing .
$$

Now $g=f^{\prime \prime}$ represents an element of $\pi_{k}\left(\mathfrak{E}\left(N^{1}, M_{m}^{1}\right.\right.$ rel $\left.\left.C\right)\right)$. This proves our claim.

Injectivity of $i_{*}$ is proved similarly.

Proof of Proposition 1. Since the total space of Lemma 3(a) is contractible, all we need to show is $\pi_{i}\left(\mathcal{E}\left(H^{1}, M_{m}^{1}\right.\right.$ rel $\left.\left.V\right)\right) \cong 0$ for all $i \geqq k$ and all $m$, because

$$
\begin{aligned}
\pi_{i+1}\left(\mathcal{E}\left(I, I \times M_{m} \operatorname{rel} 0\right)\right) & \cong \pi_{i+1}\left(\mathcal{E}\left(I \times q, M_{m}^{1} \operatorname{rel} 0 \times q\right)\right) \\
& \cong \pi_{i}\left(\mathscr{E}\left(H^{1}, M_{m}^{1} \operatorname{rel} V\right)\right)
\end{aligned}
$$

Note that

$$
\pi_{i}\left(\mathcal{E}\left(H^{1}, M_{m}^{1} \operatorname{rel} V \cup N^{1}\right)\right) \cong \pi_{i}\left(\mathscr{E}\left(L^{1}, M_{m+1}^{1} \operatorname{rel} U\right)\right)
$$

(see the next lemma and [5,3B] for a scheme of a proof). The latter group is trivial from our hypothesis if $i \leqq k$. Hence

$$
\begin{aligned}
\pi_{i}\left(\mathcal{E}\left(H^{1}, M_{m}^{1} \operatorname{rel} V\right)\right) & \cong \pi_{i}\left(\mathscr{E}\left(N^{1}, M_{m}^{1} \operatorname{rel} C\right)\right) \cong \pi_{i}\left(\mathscr{E}\left(N^{1}, M_{0}^{1} \operatorname{rel} C\right)\right) \\
& \cong \pi_{i}\left(\mathcal{E}\left(H^{1}, M_{0}^{1} \operatorname{rel} V\right)\right) \text { for } i \leqq k
\end{aligned}
$$

where the first and the last isomorphisms are obtained from the homotopy exact sequence of the fibration (b) of Lemma 3, and the second one from Lemma 5. The last group is trivial by the Alexander trick. This completes the proof.

Before starting the proof of Proposition 2, we need another lemma. Let $J=\left[0, \frac{1}{2}\right] \subset I$, and $\partial=\partial I$.

LEMMA 6. $\mathcal{E}_{1, J}\left(I, D^{n+1}\right)$ is homotopy equivalent to $\mathcal{E}_{\partial}\left(I, I \times S^{n}\right)$.

Proof. Take a small $(n+1)$-cell $E$ in $\operatorname{Int} D^{n+1}$ such that $E \cap J=J$. Then $\mathrm{Cl}\left(D^{n+1}-E\right)$ is homeomorphic to $I \times S^{n}$. Identify $\mathrm{Cl}\left(D^{n+1}-E\right)$ with $I \times S^{n}$ by this homeomorphism.

With this identification there is a natural inclusion

$$
i: \varepsilon_{\partial}\left(I, I \times S^{n}\right) \rightarrow \mathcal{E}_{1, J}\left(I, D^{n+1}\right) .
$$

Let us show that $i_{*}$ is surjective on the $k$ th homotopy group. Let 


$$
f: \Delta_{k} \times\left[\frac{1}{2}, 1\right] \rightarrow \Delta_{k} \times D^{n+1} \text { and }[f] \in \pi_{k}\left(\mathcal{E}_{1, J}\left(I, D^{n+1}\right)\right) .
$$

Then we can choose an $(n+1)$-cell $E^{\prime}$ in $\operatorname{Int} D^{n+1}$ such that $E^{\prime} \cap J$ $=J$ and $E^{\prime} \cap \operatorname{Im} f=\Delta_{k} \times \frac{1}{2}$. We can find a $k$-modification $h$ of $D^{n+1}$ keeping $\partial D^{n+1} \cup I$ fixed such that $h_{0}=$ identity and $h_{1}\left(E^{\prime}\right)=E$. Now $h_{1} \circ f \in \mathcal{E}_{\partial}\left(I, I \times S^{n}\right)$. This proves the surjectivity. Injectivity is proved similarly.

2. Transverse intersection. In this section we start a proof of Proposition 2. Because there is a Kan fibration

$$
\mathcal{E}_{1, J}\left(I, D^{n+1}\right) \hookrightarrow \mathcal{E}_{0,1}\left(I, D^{n+1}\right) \stackrel{\ulcorner}{\hookrightarrow} \mathcal{E}_{0}\left(J, D^{n+1}\right),
$$

where the total space is contractible by the Alexander trick, and the fibre is homotopy equivalent to $\varepsilon_{\partial}\left(I, I \times S^{n}\right)$, it is sufficient to show that

$$
\pi_{k}\left(\mathcal{E}_{0}\left(J, D^{n+1}\right)\right) \stackrel{e_{*}}{=} \pi_{k}\left(S^{n}\right) \quad \text { for } k \leqq p+2 .
$$

In order to define $e$, let $f: \Delta_{k} \times J \rightarrow \Delta_{k} \times D^{n+1}$ represent a $k$ th homotopy group element; then the composition

$$
\Delta_{k} \times \frac{1}{2} \stackrel{f}{\longrightarrow} \Delta_{k} \times\left(D^{n+1}-0\right) \rightarrow D^{n+1}-0 \rightarrow S^{n}
$$

represents $e_{*}([f])$.

It is easy to see that $e_{*}$ is surjective. We show that $e_{*}$ is injective. For this purpose, assume that $e_{*}([f])=0$.

The symbol $\Delta$ stands for $\Delta_{k}$ when no confusion is expected.

Now choose a number $d(0<d<1)$, so that $f(x \times \operatorname{Int} J) \cap x \times d S^{n} \neq \varnothing$ for all $x \in \Delta$.

Then choose triangulations of $\Delta \times J, \Delta \times D^{n+1}$ and $\Delta$ so that the following conditions are satisfied.

(1) $f$ is a simplicial map with respect to these triangulations.

(2) $\pi: \Delta \times D^{n+1} \rightarrow \Delta$ is simplicial.

(3) $\Delta \times d S^{n}, \operatorname{Im} f \cap \Delta \times d S^{n}, \Delta \times 0$ are subcomplexes. The triangulation of $\Delta$ above is denoted by $C$. Also, if $S \subset \Delta \times D^{n+1}$ and $B \subset \Delta, S \mid B=S$ $\cap \pi^{-1}(B)$.

We will look at $K=\operatorname{Im} f \cap \Delta \times d S^{n}$ first.

Definition. If $\sigma$ is a simplex of $e$, we denote components of $\left.K\right|_{\text {Int } \sigma}$ by $C(\dot{\boldsymbol{\sigma}})_{1}, \ldots, C(\dot{\boldsymbol{\sigma}})_{m}$ (for some $\left.m\right)$. We order $C(\dot{\boldsymbol{\sigma}})_{1}, C(\dot{\boldsymbol{\sigma}})_{2}, \ldots, C(\dot{\boldsymbol{\sigma}})_{m}$ so that $p_{2}\left(f^{-1}\left(C(\dot{\sigma})_{i} \mid x\right)\right)<p_{2}\left(f^{-1}\left(C(\dot{\sigma})_{j} \mid x\right)\right)$ if and only if $i<j$ for $x \in \operatorname{Int} \sigma$, where $p_{2}$ is the projection $\Delta \times J \rightarrow J$. The closure of $C(\dot{\sigma})_{i}$ is denoted by $C(\sigma)_{i}$.

Note that $\pi\left(C(\sigma)_{i}\right)=\sigma$ and $C(\sigma)_{i}$ is a simplex in $K$.

Lemma 7. We may assume that $\operatorname{dim}\left(C(\sigma)_{i}\right)=\operatorname{dim} \sigma$, hence $\pi: C(\sigma)_{i} \rightarrow \sigma$ is a homeomorphism.

This is a direct consequence of the general position lemma ([6, Lemma 3.4], 
or [2], for example) which states that there is a $k$-modification $F$ of $D^{n+1}$ such that

$$
\operatorname{Im}\left(F_{1} \circ f\right) \cap x \times d S^{n} \leqq \max \left(0, \operatorname{dim} J+\operatorname{dim}\left(d S^{n}\right)-\operatorname{dim} D^{n+1}\right) .
$$

Definition. (1) When $a \in C(\sigma)_{i}$ with $\operatorname{dim} \sigma=k$, and $f$ intersects Int $\sigma$ $\times d S^{n}$ transversely along $C(\dot{\sigma})_{i}$, we call the point a essential. Otherwise it is removable.

$$
\begin{aligned}
M_{f}^{\prime} & =\bigcup_{x \in \Delta}\left\{f(x, p) \mid p=\min _{q \in J}\left\{q \mid f(x, q) \in x \times d S^{n}\right\}\right\}, \\
M_{f} & =\mathrm{Cl}\left(M_{f}^{\prime}\right) .
\end{aligned}
$$

Note that $M_{f}=\cup_{\sigma \in \mathcal{C}} C(\sigma)_{1}$, hence it is a subcomplex of $K$.

Lemma 8. There exists a k-modification $F$ such that the subcomplex $M_{F_{1} \circ f}$ defined by using $F_{1} \circ f$ (instead of $f$ ) consists of essential points only.

For the proof we would like to define a $k$-modification by the join extension which will be useful quite of ten.

LEMMA 9. Let $E$ be an $(n+k+1)$-cell in $\Delta \times\left(D^{n+1}-0\right)$ such that $\sigma=\pi(E)$ is a $k$-cell, and let $Y$ be a convex $n$-cell in $D^{n+1}$ so that if $W=\sigma \times Y, E \cap W$ is a properly embedded $(k+n)$-cell in $E$. If $X=(\partial E \cap W) * b$, where $b$ $\in$ Int $E$, then there is a $k$-modification $F$ of $D^{n+1}$ such that

(i) $F_{1}(W \cap E)=X$,

(ii) $F_{t} \mid \Delta \times D^{n+1}-E=$ identity .

Note that if $E \subset I \times D^{n}, Y=d \times D^{n}, b=d^{\prime} \times y\left(0<d^{\prime}<d\right)$, and

$$
\operatorname{Im} f \cap x \times Y \cap E=\operatorname{Im} f \cap x \times[d, 1] \times D^{n} \cap E=\{\text { a point }\}
$$

for $x \in \operatorname{Int} \pi(E)$, in addition, then $F_{1}(\operatorname{Im} f) \cap x \times Y \cap E=\varnothing$ for $x$ $\in \operatorname{Int} \pi(E)$.

Note also that even if $Y^{\prime}$ is not convex, we can still apply the above argument to push $\operatorname{Im} f \cap x \times Y^{\prime} \cap E$ off $x \times Y^{\prime}$ as long as there is a homeomorphism commuting with the projection of pairs between $\left(E, \sigma \times Y^{\prime}\right.$ $\cap E)$ and $(E, W \cap E)$, with $W$ convex.

Proof of LEMMA 8. This is an application of the join modification defined above. We start from $k$-simplices of $\mathcal{C}$ and work downward. Let $\sigma \in \mathcal{C}$ be a $k$ simplex, then from our choice of $d$ in the beginning and Lemma 7 (application of general position), we find $C(\dot{\sigma})_{m}$, where $m$ is the smallest of $i$ 's such that $C(\dot{\sigma})_{i}$ consists of transverse intersection points. If $m=1$, we have the situation we want. Suppose $m>1$, then $C(\dot{\sigma})_{1}$ is removable. It is not difficult to find an $(n+k+1)$-cell $E$ in $\Delta \times\left(D^{n+1}-0\right)$ that satisfies the following conditions:

(1) $\pi(E)=\sigma$.

(2) $E \cap f(x \times J) \cap x \times d D^{n+1}$ is a properly embedded 1-disk in $\left.E\right|_{x}$ for all $x \in \operatorname{Int} \sigma$. 
(3) $\left.E\right|_{x}=\left.C(\sigma)_{1}\right|_{x}$ if $x \in \partial \sigma$.

(4) If $E \cap C(\dot{\sigma})_{i} \neq \varnothing$, then $i=1$.

(5) $\left(\left.E\right|_{x}, E \cap x \times d S^{n}\right)$ is homeomorphic to the standard disk pair $\left(D^{n+1}\right.$, $\left.D^{n}\right)$ for $x \in \operatorname{Int} \sigma$.

Now push $C(\dot{\sigma})_{1}$ off $x \times d S^{n}(x \in \operatorname{Int} \sigma)$ by the previous lemma, by choosing $b=v \times w$ where $v$ is the barycenter of $\sigma$ and

$$
w \in \operatorname{Int}\left(E \cap v \times d D^{n+1}\right) .
$$

By Lemma 9 this can be realized as a $k$-modification. Repeat the process until all $C(\sigma)_{i}$ 's with $i<m$ are removed.

We repeat the process for all $k$-simplices of $\varrho$. Suppose we have removed the removable points as above over all simplices which are $(j+1)$-dimensional or higher.

Let $\sigma$ be a $j$-simplex of $\mathcal{C}$, and assume $C(\sigma)_{1}$ is removable as before.

If we let $N(\sigma, \Delta)$ denote a $k$-cell in $\Delta$ which is a relative neighborhood of $\sigma$ in $\Delta$, we can construct an $(n+k+1)$-cell $E$ satisfying similar conditions as above by taking $N(\sigma, \Delta)$ small enough. Choosing $b=v \times w$, where $v$ is the barycenter of $\sigma$ and $w$ as before, we can remove $C(\sigma)_{1}$ by the join modification. The join modification does not produce new intersection of $\operatorname{Im} f$ and $\Delta \times d S^{n}$ because of its definition. Repeating the process until $j=0$, we are done.

If we assume that our original $f$ satisfies the conclusion of Lemma 8 , we can conclude that

$$
\begin{gathered}
T \subset \Delta \times d D^{n+1}, \\
T \cap \Delta \times d S^{n}=\bigcup_{i=1}^{N} C\left(\sigma_{i}\right)_{1}=M_{f},
\end{gathered}
$$

where

$$
T=T_{f}=\bigcup_{x \in \Delta} f(x \times[0, t(x)]), \quad t(x)=\max \left\{t^{\prime}(x) \mid f\left(x \times t^{\prime}(x)\right) \in M_{f}\right\},
$$

and $\sigma_{1}, \ldots, \sigma_{N}$ are all $k$-simplices of $\mathcal{C}$. We use $T_{x}=T \cap \pi^{-1}(x)$ in the rest of the paper.

3. Proof of Proposition 2. We continue the proof of Proposition 2 started in the previous section. In particular, we assume that the representative $f$ satisfies conditions (a) and (b) of $\$ 2$ as well as conditions (1), (2) and (3) in the beginning of the section.

Our objective is to prove

Lemma 10. Suppose $\pi_{i}\left(\mathcal{E}_{0}\left(I, I \times M_{n}\right)\right) \cong 0$ for $i \leqq k-2$. Then there exists a $k$-isotopy $g \in \mathcal{E}_{0}\left(-d I, d D^{n+1}\right)$ satisfying

(a) $\operatorname{Im} g \cap T=\Delta \times 0$.

(b) $g \mid \partial \Delta \times(-d I) \cup \Delta \times 0=$ identity.

Note that a new $k$-isotopy 


$$
f \cup g: \Delta \times[-d, \delta] \rightarrow \Delta \times d D^{n+1}
$$

defined by

$$
f \cup g \mid \Delta_{k} \times \delta I=f \text { and } f \cup g \mid \Delta_{k} \times(-d I)=g
$$

for some $\delta(0<\delta<d)$ represents an element of $\pi_{k}\left(\varepsilon_{0}\left([-d, \delta], d D^{n+1}\right)\right)$.

Proof of Proposition 2 assuming Lemma 10. In order to show that the homomorphism $e_{*}$ defined in $\$ 2$ is injective, it is sufficient to show that $\gamma_{*}([f])=0$. Here

$$
\bigcup_{J^{\prime} \subset J} \mathcal{E}_{J^{\prime}}\left(J, D^{n+1}\right) \hookrightarrow \mathcal{E}_{0}\left(J, D^{n+1}\right) \stackrel{\gamma}{\rightarrow} G \mathcal{E}_{0}\left(J, D^{n+1}\right)
$$

is a Kan fibration in which $\gamma$ is to take germs near 0. (See [1], for example.)

Lemma 4 and a method similar to the proof of Lemma 6 or $[5,3 \mathrm{~A}]$ show that the fibre is contractible, and imply our assertion above.

Let $f: \Delta \times J \rightarrow \Delta \times D^{n+1}$ be as before. If we take a small enough positive $\delta<1$, then

$$
f(\Delta \times[0, \delta]) \subset \Delta \times \operatorname{Int} D^{n+1} \text { and } f(\Delta \times[0, \delta]) \cap \operatorname{Im} g=\Delta \times 0 .
$$

It is sufficient to show that the element of $\pi_{k}\left(\varepsilon_{0}\left([0, \delta], D^{n+1}\right)\right)$ represented by the restriction of $f$ is trivial when

$$
h=f \mid \Delta \times \delta: \Delta \times \delta \rightarrow \Delta \times\left(d D^{n+1}-0\right) \rightarrow d S^{n}
$$

represents the trivial element of $\pi_{k}\left(d S^{n}\right) \cong \pi_{k}\left(S^{n}\right)$. First, we may assume that $g \mid \Delta \times(-d)=$ identity. Indeed, consider the Kan fibration

$$
\mathcal{E}\left(\left\{a_{1}, a_{2}\right\}, S^{n} \text { rel }\left\{a_{1}\right\}\right) \hookrightarrow \mathcal{E}\left(\left\{a_{1}, a_{2}\right\}, S^{n}\right) \stackrel{r}{\longrightarrow} \mathcal{E}\left(a_{1}, S^{n}\right),
$$

where the fibre is contractible.

Now the $k$-isotopy $f \cup g$ defined in the lemma can be extended to $H \in \mathcal{H}\left(d D^{n+1}\right.$ rel 0$)$. We can require that $H \mid \partial \Delta \times d D^{1}=$ identity by the Alexander trick. Hence $H \mid \Delta \times\{-d, d\}$ represents an element of

$$
\pi_{k}\left(\mathcal{E}\left(\left\{a_{1}, a_{2}\right\}, S^{n}\right)\right)
$$

by a suitable identification. Since $r_{*}$ is an isomorphism, this element is trivial if and only if $H \mid \Delta \times d$ is. Using $H(\Delta \times[\delta, d])$ as the trace of homotopy, we see that this represents the trivial element if $f \mid \Delta \times \delta$ represents the trivial element in $\pi_{k}\left(S^{n}\right)$.

Now $f \cup g$ represents an element of $\pi_{k}\left(\mathscr{E}\left(-I \cup J, D^{n+1}\right.\right.$ rel $\left.\left.\{-1,0\}\right)\right)$ by an identification $\left(d D^{n+1}\right.$ is identified with $D^{n+1},-d I$ with $-I$, and $[0, \delta]$ with $\left.J\right)$. We show that this is trivial. Note that in the following Kan fibrations

$$
\begin{aligned}
& \mathcal{E}\left(-I \cup J, D^{n+1} \operatorname{rel}\{-1,0\}\right) \subset \mathcal{E}\left(-I \cup J, D^{n+1} \operatorname{rel}(-1)\right) \stackrel{r}{\rightarrow} \mathcal{E}\left(0, D^{n+1}\right), \\
& \mathcal{E}\left(-I \cup J, D^{n+1} \operatorname{rel}\left\{-1, \frac{1}{2}\right\}\right) \subset \mathcal{E}\left(-I \cup J, D^{n+1} \operatorname{rel}(-1)\right) \stackrel{r}{\rightarrow} \mathcal{E}\left(\frac{1}{2}, D^{n+1}\right),
\end{aligned}
$$


where both $r$ 's are restrictions, the base spaces are contractible. So is $\mathcal{E}\left(-I \cup J, D^{n+1}\right.$ rel $\left.\left\{-1, \frac{1}{2}\right\}\right)$ by the Alexander trick. This shows

$$
\mathcal{E}\left(-I \cup J, D^{n+1} \operatorname{rel}\{-1,0\}\right)
$$

is also contractible. This shows, in particular, that $f \mid \Delta \times[0, \delta]$ represents the trivial element in $\pi_{k}\left(\mathscr{E}_{0}\left([0, \delta], D^{n+1}\right)\right)$.

Proof of Lemma 10. We have chosen the triangulations so that $f$ and the projection $\pi: \Delta_{k} \times d D^{n+1} \rightarrow \Delta_{k}$ are simplicial. The construction of $g$ is done skeletonwise starting from the 0 -skeleton $\Delta_{k}^{(0)}$ where it is obvious because $\left(d D^{n+1}-\operatorname{Im} f\right) \cup\{0\}$ is connected. $\left(g \mid \partial \Delta_{k} \times(-d I)\right.$ is defined to be the identity once and for all.)

Suppose we have constructed $g$ on $\Delta_{k}^{(j-1)} \times(-d I)$. Let $\sigma$ be a $j$-simplex of $\Delta_{k}$ with the above triangulation. Then $g$ can be extended to $N(\partial \sigma) \times(-d I)$, where $N(\partial \sigma)$ is a collar of $\partial \sigma$ in $\sigma$. To see this, first note that for a small $\varepsilon>0$ there is a $j$-homeotopy $F \in \mathcal{H}\left(d D^{n+1}\right.$ rel $\left.d S^{n} \cup\{0\}\right)$ such that

$$
F(x \times[0, \varepsilon])=f(x \times[0, \varepsilon]) \subset x \times \operatorname{Int} d D^{n+1}
$$

for all $x \in \sigma$, by the $j$-isotopy extension theorem of Hudson ([3] or [4]) and our codimension restriction. Let $P=F^{-\mathrm{i}}(g(\partial \sigma \times(-d I)))$, and $L$ be a subpolyhedron of $(\partial \sigma \times I) \times d D^{n+1}$ defined as $\cup_{x \in \partial \sigma ; t \in I}\left(x, t, F\left(P_{x}\right)\right)$, where $N(\partial \sigma)$ is identified with $\partial \sigma \times I$ and $x \in \partial \sigma=\partial \sigma \times 0$. If we take $N(\partial \sigma)$ small enough, $L \cap T_{x}=x \times 0$ for any $x \in N(\partial \sigma)$. Define $g(y \times(-d I))=L_{y}$ for $y$ $\in N(\partial \sigma)$. The actual definition of $g$ is easy because $\mathcal{H}_{\partial}\left(D^{1}\right)$ is contractible. Let $K=T \cap \pi^{-1}\left(x_{0}\right)$. Without loss of generality we may assume that $K \cap$ $x_{0} \times(-d I)=x_{0} \times\{0\}$ by moving $K$ via an isotopy if necessary.

Note that for any $j$-cell $\sigma^{\prime}$ in $\sigma\left(x_{0} \in \sigma^{\prime}\right.$ and $\left.\sigma \in \mathcal{C}\right)$, there is a $j$-homeotopy between $\sigma^{\prime} \times K$ and $T \cap \pi^{-1}\left(\sigma^{\prime}\right)$ because $f$ was simplicial with respect to $C$ and we assume condition (b) of $\S 2$ is satisfied, where $\sigma^{\prime}=\mathrm{Cl}(\sigma-N(\partial \sigma))$. We can choose $N(\partial \sigma)$ so that $\sigma^{\prime}$ is a convex cell. By a similar application of the isotopy extension theorem of Hudson again, we identify $T \cap \pi^{-1}\left(\sigma^{\prime}\right)$ with $\sigma^{\prime} \times K$. Then we can consider $g \mid \partial \sigma^{\prime} \times(-d I)$ to represent an element in $\pi_{j-1}\left(\mathscr{E}\left((-d I) \cup K, d D^{n+1} \operatorname{rel} K\right)\right)$.

Because $n \geqslant 3$, we can find a manifold $N$ in $d D^{n+1}$ such that

(i) $K-0 \subset \operatorname{Int} N \cup\left(N \cap d S^{n}\right)$,

(ii) $\mathrm{Cl}\left(d D^{n+1}-N\right) \approx I \times M_{\nu}$ for some $\nu$,

(iii) $\operatorname{Im} g \cap x \times N=x \times 0$ for $x \in \partial \sigma^{\prime}$.

Now $g \mid \partial \sigma^{\prime} \times(-d I)$ represents an element of $\pi_{j-1}\left(\mathcal{E}\left(I, I \times M_{\nu}\right.\right.$ rel 0$\left.)\right)$ by this identification. The hypothesis of Lemma 10 claims this is trivial if $j \leqq k-1$, and $g$ can be extended to $\sigma^{\prime} \times(-d I)$. Since the extension is done as an element of $\mathcal{E}\left(I, I \times M_{\nu}\right.$ rel 0$)$, all the conditions of the lemma are satisfied.

This argument can be used as long as $j \leqq k-1$, because our hypothesis is valid in this range. Assume that we have constructed $g$ on $\Delta^{(k-1)} \times(-d I)$. Let $\sigma$ be a $k$-simplex of $\Delta$ (with the same triangulation $\mathcal{C}$ ). As before, we can extend 
$g$ to $N(\partial \sigma) \times(-d I)$. Again as before, we may assume $T \cap \pi^{-1}\left(\sigma^{\prime}\right)=\sigma^{\prime} \times K$. However, by Lemma 8 we know that $\left(x \times d D^{n+1}, T_{x}\right) \approx\left(D^{n+1}, I\right)$ and we may assume $T \cap \pi^{-1}\left(\sigma^{\prime}\right)=\sigma^{\prime} \times d I$. Now $g \mid \partial \sigma^{\prime} \times(-d I)$ represents a $(k$ - 1)st homotopy group element of $\mathcal{E}\left(-d I \cup d I, d D^{n+1}\right.$ rel $\left.d I\right)$. Since this complex is contractible by the Alexander trick, the extension is complete.

\section{BIBLIOGRAPHY}

1. T. Akiba, Homotopy groups of $\&\left(S^{n}, S^{n+r}\right)$, Michigan Math. J. 21 (1974), 371-376.

2. - Filling in cross sections of PL-bundles (to appear).

3. J. F. P. Hudson, Extending piecewise-linear isotopies, Proc. London Math. Soc. (3) 16 (1966), 651-668. MR 34 \#2020.

4. - Concordance, isotopy and diffeotopy, Ann. of Math. (2) 91 (1970), 425-448. MR 41 \#4549.

5. N. H. Kuiper and R. K. Lashof, Microbundles and bundles. I, II, Invent. Math. 1 (1966), 1-17; 243-259. MR 35 \#3339; 35 \#7340.

6. K. C. Millett, Piecewise linear concordance and isotopies, Mem. Amer. Math. Soc. No. 153 (1974), 1-73.

7. C. P. Rourke and B. J. Sanderson, Introduction to piecewise-linear topology, Ergebnisse Math. Grenzgebiete, Band 69, Springer-Verlag, New York, 1972. MR 50 \#3236.

Department of Mathematics, Tufts University, Medford, Massachusetts 02155 